\title{
Successful use of eculizumab to treat atypical hemolytic uremic syndrome in patients with inflammatory bowel disease
}

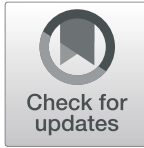

Ramy M. Hanna ${ }^{1,2^{*}}$ (D), Noah Merin ${ }^{3}$, Richard M. Burwick' ${ }^{4}$ Lama Abdelnour ${ }^{1}$, Umut Selamet ${ }^{1}$, Beshoy Yanny ${ }^{5}$, Patrick Bui ${ }^{6}$, Mary Fouad ${ }^{6}$ and Ira Kurtz ${ }^{1,7}$

\begin{abstract}
Background: Atypical hemolytic uremic syndrome is a rare group of disorders that have in common underlying complement amplifying conditions. These conditions can accelerate complement activation that results in a positive feedback cycle. The known triggers for complement activation can be diverse and include, infection, autoimmune disease, and malignancy. Recent reports suggest that certain autoimmune and rheumatological triggers of complement activation may result in atypical hemolytic uremic syndrome that does not resolve despite treating the underlying disorder. Specifically, patients with systemic lupus erythematosus and microangiopathic hemolysis may not respond to treatment of their underlying rheumatological trigger but responded to complement blockade.
\end{abstract}

Case presentations: We report two patients with inflammatory bowel disease complicated by development of atypical hemolytic uremic syndrome. In both cases, patients were on treatment for inflammatory bowel disease, that was not well controlled/flaring at the time. The first patient is a male who developed Crohn's disease and microangiopathic hemolysis at age 5 and was treated with eculizumab successfully. Discontinuation of the medication led to multiple relapses, and the patient currently is being treated with eculizumab and has normal hematological and stable renal parameters. The second patient is a 49-year-old female with Ulcerative Colitis treated with 6-Mercaptopurine. She developed acute kidney injury and microangiopathic hemolysis. Prompt diagnosis and treatment with eculizumab resulted in the recovery of kidney injury along with a complete hematological response.

Conclusions: These two cases are the fifth and sixth patients to be published in the literature with atypical hemolytic uremic syndrome and inflammatory bowel disease treated with complement blockade. This confirms that C5 complement blockade is effective in treating complement mediated thrombotic microangiopathy/atypical hemolytic uremic syndrome when it is triggered in patients with inflammatory bowel disease.

Keywords: Atypical hemolytic uremic syndrome, Complement, Complement blockade, Thrombotic Microangiopathy, Inflammatory bowel disease, Crohn's colitis/Crohn's disease, Ulcerative colitis

\footnotetext{
* Correspondence: rhannamd81@yahoo.com; rmhanna@mednet.ucla.edu ${ }^{1}$ Department of Medicine, Division of Nephrology, UCLA David Geffen School of Medicine, Room 7-155, Factor Bldg. 700 Tiverton Ave, Los Angeles, CA 90095, USA

${ }^{2}$ Department of Medicine, Division of Nephrology, University of California

Irvine School of Medicine, Irvine CA, USA

Full list of author information is available at the end of the article
}

(c) The Author(s). 2019 Open Access This article is distributed under the terms of the Creative Commons Attribution 4.0 International License (http://creativecommons.org/licenses/by/4.0/), which permits unrestricted use, distribution, and reproduction in any medium, provided you give appropriate credit to the original author(s) and the source, provide a link to the Creative Commons license, and indicate if changes were made. The Creative Commons Public Domain Dedication waiver (http://creativecommons.org/publicdomain/zero/1.0/) applies to the data made available in this article, unless otherwise stated. 


\section{Key points}

1) IBD can trigger aHUS, and in both cases, we report patients received eculizumab and other biologic agents for IBD concurrently.

2) We report the 5th and 6th cases of complement mediated TMA/aHUS in IBD patients (1 with UC and 1 with $\mathrm{CD}$ ), with successful resolution of aHUS with complement blockade.

3) We report prevention of relapse with ongoing complement blockade in one case.

\section{Background}

Atypical Hemolytic Uremic Syndrome (aHUS), is a syndrome resulting from complement activation. The causes of complement activation are numerous, and the genetics are diverse. In affected patients, a latent susceptibility becomes unmasked by a complement amplifying condition resulting in Thrombotic Microangiopathy (TMA) [1]. New research is revealing complement biology as having a role in microangiopathic hemolysis and endothelial dysfunction in many rheumatological diseases [1]. Recent publications by Park et al. disclose that ongoing microangiopathic hemolysis stemming from rheumatological conditions like systemic lupus erythematosus can be treated with eculizumab when more traditional immunosuppression has failed [2, 3]. Microangiopathic hemolysis due to Antiphospholipid antibody syndrome and catastrophic antiphospholipid antibody syndrome (CAPS) variant has also been successfully treated with complement blockade [4].

The final common pathway of a positive feedback complement activation loop is the etiologic feature of aHUS, despite the various triggers [1]. In most cases, a strong genetic susceptibility is unmasked by the presence of a strong temporary, or a milder but lingering complement amplifying condition [1]. Indeed, some cases of aHUS can be self-limiting (due to hypertension, post-partum aHUS), and others can be due to slow ongoing complement activation (C3 glomerulopathy, and chronic hypertension due to occult TMA) [1].

Inflammatory bowel disease (IBD), resulting in Crohn's Disease (CD) and Ulcerative Colitis (UC) as clinical phenotypes, is one such group of complement amplifying conditions [5]. As with other autoimmune diseases, firstline treatment has always focused on suppression of the immune system to eliminate inflammation and clinical symptoms. In the current literature, there are three published case reports and one abstract detailing patients with IBD and aHUS treated with eculizumab [6-10].

We report two additional cases of patients with IBD and aHUS treated with eculizumab. Patient 1 is a 19year-old male with $\mathrm{CD}$ who was treated with eculizumab at age 5 and had multiple relapses off the drug. He was maintained on eculizumab with near-normal renal and hematological parameters for 14 years. Patient 2 is a 49 year-old female with UC who developed severe aHUS and was promptly treated with eculizumab resulting in normalization of renal and hematological parameters.

\section{Case presentations}

\section{Patient 1}

The first patient is a 19-year-old Caucasian male who presented for the continuation of complement blockade therapy with eculizumab. He was diagnosed with $C D$ at age 5 and started on per rectum steroid therapy with budesonide and mesalamine therapy. He did require continuous infusions of adalimumab then infliximab for control of his disease. During the initial episode of colitis/CD in 2005, he was noted to have thrombocytopenia, hemolytic anemia, schistocytes with elevations in lactate dehydrogenase. The ADAMTS13 level was not available early on in the time of his diagnosis. Though Shiga toxin was not found in stool, vitamin B12 levels was 781 nanogram $(\mathrm{ng}) / \mathrm{mL}$, red blood cell (RBC) folate levels were within normal limits 981 nanomoles/L excluding other known TMA causes at that time. Rheumatolgoic serologies like anti nuclear antibody (ANA), anti double stranded DNA (dsDNA), anti ribonucleic protein smith (RNP-smith), anti cardiolipin (anti CL), dilute russell viper venom time assay (DRVVT), and anti phospholipid antibody (anti APL $\mathrm{Ab})$ studies were all negative during initial workup.

As the scientific understanding of aHUS developed, it was decided that eculizumab would be initiated on an experimental basis with institutional review board approval (in a health system in Florida). He underwent a several-month course of eculizumab with clinical response. Following discontinuation of the medication, the patient suffered a relapse a few months later. Therapy was reinitiated and was subsequently weaned off successfully. Complement testing found a high level of C5 convertase function with high soluble membrane attack complex that improved with eculizumab use.

In April 2010 the patient had a severe flare of his underlying $\mathrm{CD}$ despite ongoing use of adalimumab, his disease remained poorly controlled clinically. He had a concomitant serum creatinine elevation peaking at 3.13 $\mathrm{mg} / \mathrm{dL}$ in $04 / 2010$ (baseline of $0.57 \mathrm{mg} / \mathrm{dL} 08 / 2009$ ) with a drop in hemoglobin from $12.9 \mathrm{~g} / \mathrm{L}$ baseline in $08 / 2009$ to $7.6 \mathrm{~g} / \mathrm{L}$ in $03 / 2010$. The total bilirubin concentration was not elevated but increased from a baseline of 0.1 $(\mathrm{mg} / \mathrm{dL})$ in $03 / 2010$ to $0.8(\mathrm{mg} / \mathrm{dL})$ in $04 / 2010$ during the flare. Lactate dehydrogenase (LDH) was significantly elevated at 2223 Units/L in 4/2010 and decreased after that. The platelets had decreased by $\sim 50 \%$ from 366 , $000 / \mathrm{uL}$ at baseline in $02 / 2010$ to $187,000 / \mathrm{uL}$ in $4 / 2010$. ADAMTS 13 activity level was within normal limits at 
44\% ruling out ADAMTS13 deficiency and thrombotic thrombocytopenic purpura (TTP). Prothrombin time (PT) was $11 \mathrm{~s}$ with an International Normalized Ratio (INR) was 1, and activated partial thromboplastin time (aPTT) was $39 \mathrm{~s}$ both within normal. Fibrinogen levels were high at $471 \mathrm{mg} / \mathrm{dL}$, and a D-dimer was high at 6.17 microgram $/ \mathrm{mL}$ as expected in an inflammatory condition. Absolute reticulocyte count was in the upper limit of normal / slightly elevated at $2.92 \%$.

Since the patient had an established aHUS diagnosis, eculizumab was promptly reintroduced at $900 \mathrm{mg}$ (infusion dose) each week with normalization of the platelet count and LDH levels, and improvement of the serum $\mathrm{Cr}$ to $1 \mathrm{mg} / \mathrm{dL}$. The patient received eculizumab (1200 mg infusions) every two weeks for 8.5 years continually after this aHUS relapse. The patient had already received appropriate meningococcal vaccinations from prior courses and was aware of the risks and benefits of pharmacological complement blockade. He has not had any issues with meningitis or other infections linked to eculizumab. Clinically, his IBD symptoms slowly improved with use of ongoing adalimumab and steroid therapy for $\mathrm{CD} / \mathrm{IBD}$ along with eculizumab use for aHUS.

The effect of this regimen was the maintenance on his aHUS was the maintenance of normal hematological parameters and renal function with current serum $\mathrm{Cr}$ of 1 $\mathrm{mg} / \mathrm{dL}$, hemoglobin of $10-12 \mathrm{~g} / \mathrm{L}$, and LDH within normal limits. The patient has agreed to continue lifelong eculizumab in this case given his two prior relapses. No antibodies against complement factors were found, and no mutations were found. Only $60 \%$ of patients with aHUS have detectable mutations, and in some cases, the mutations can be present but are currently unknown [11]. Figure 1 details laboratory findings in patient 1 and his course during his last flare in 2010.

\section{Patient 2}

The second patient is a 49-year-old Caucasian female with a history of UC treated with six mercaptopurine (6MP), and steroids per rectum [budesonide]. She developed a flare of ulcerative colitis symptoms, along with increased platelet count from 322,000 platelets/ $\mathrm{uL}(09 / 2018)$ to 622,000 platelets/uL (10/2018). This was a reactive thrombocytosis that occurred initially, and was clinically concomitant with a flare despite treatment with budesonide and 6MP.Her serum $\mathrm{Cr}$ at baseline was $0.51 \mathrm{mg} / \mathrm{dL}$, and her hemoglobin was $13.1 \mathrm{~g} / \mathrm{L}$. She presented to the emergency department one month after the start of this flare with weakness and was found to have a decreased hemoglobin concentration of $4.3 \mathrm{~g} / \mathrm{L}$, serum $\mathrm{Cr}$ of $3.2 \mathrm{mg} / \mathrm{dL}, \mathrm{LDH}$ of 1000 units/L, and schistocytes on the peripheral smear. Her platelets had droped from baseline of 322, 000 platelets/ul and more recent peak of 622,000 platelets/ul to 83,000 platelets/ul; this level dropped further to 43,000 plateltes/ul at a nadir 11/17/2018. These findings and the hemolysis suggested thrombotic microangiopathy like aHUS as the likely diagnosis over 6-mercaptopurine bone marrow toxicity which was another possibility. Vitamin B12 level was normal at $769 \mathrm{ng} / \mathrm{mL}$, serum folate level was also

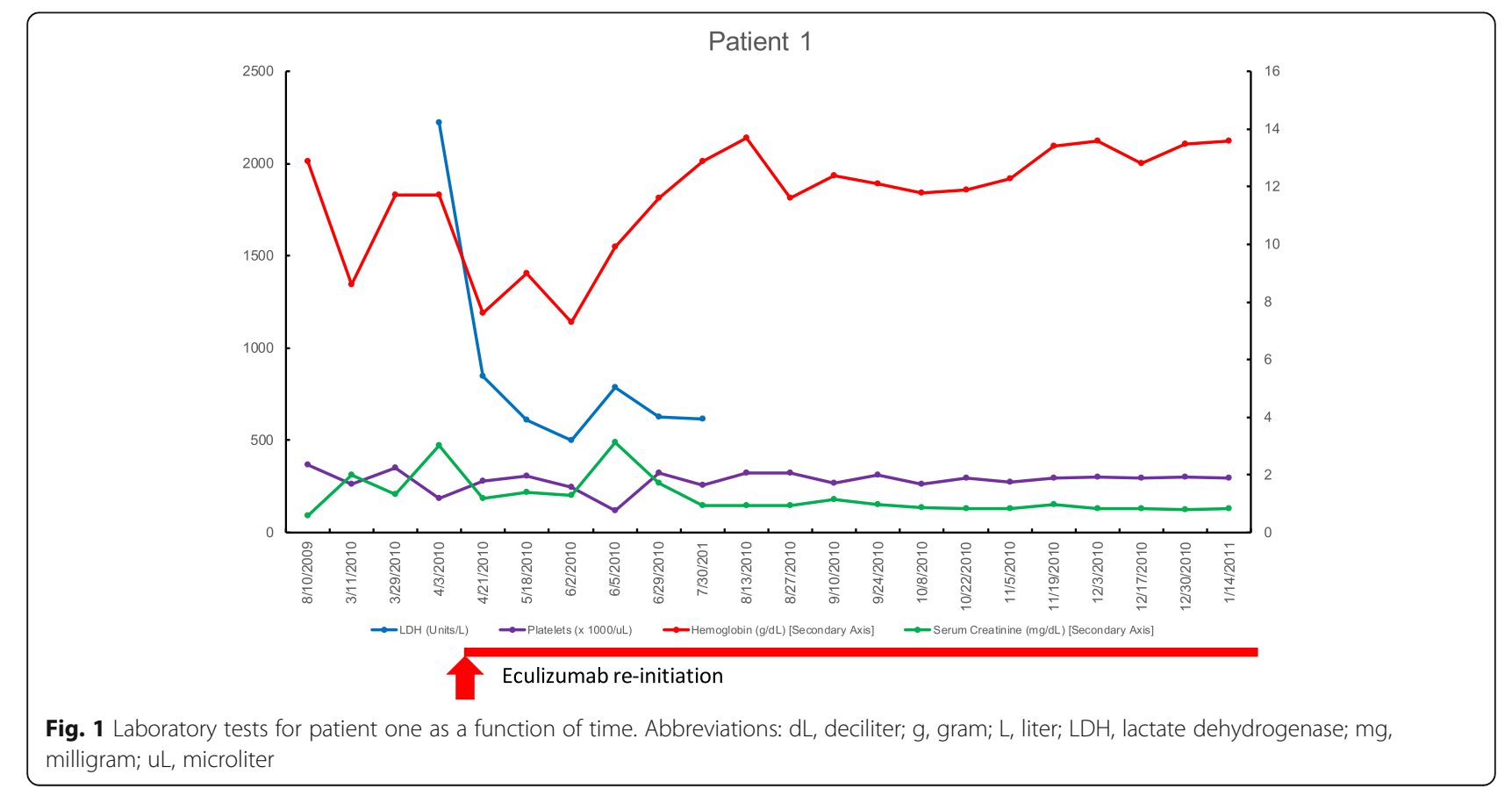




\section{Patient 2}

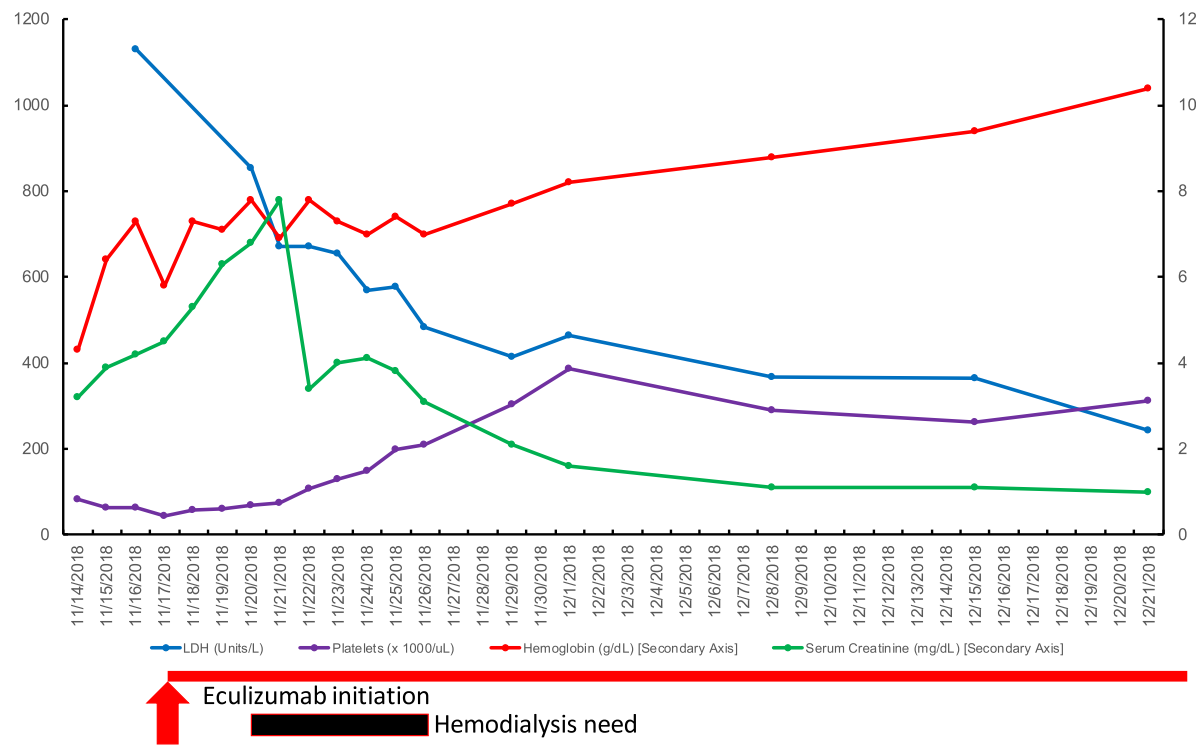

Fig. 2 Laboratory results for patient two as a function of time. Abbreviations: dL, deciliter; g, gram; L, liter; LDH, lactate dehydrogenase; mg, milligram; $\mathrm{UL}$, microliter

normal at $12.9 \mathrm{ng} / \mathrm{mL}$. PT was $13 \mathrm{~s}$ with an INR of 1.3, aPTT was slightly elevated at $39 \mathrm{~s}$. Fibrinogen levels were high normal range at $313 \mathrm{mg} / \mathrm{dL}$, with an elevated D-dimer 3.28 microgram $/ \mathrm{mL}$. Rheumatologic serologies (ANA, anti dsDNA, anti RNP sm, anti CL, DRVVT, APL Ab) were all negative. Reticulocyte counts were obtained at $2 \%$ (normal), and one result was $3 \%$ which is slightly elevated, and not expected in bone marrow toxicity.

The finding of an ADAMTS13 level of $80.8 \%$ suggested that the cause of TMA was not TTP. The patient was transferred back to a quaternary care center, and then eculizumab was started after meningococcal vaccination. The patient agreed to the risks and benefits of complement blockade in the absence of waiting the requisite two weeks for development of meningococcal antibody titers after immunization, and antibiotic prophylaxis with penicillin-based antibiotics was provided.

The serum Cr, platelets, hemoglobin, LDH all improved quickly and normalized (Fig. 2). The patient required hemodialysis for $\sim 1$ week and was weaned off. Eculizumab was administered at a given dose of $900 \mathrm{mg}$ weekly infusions. The patient was discharged on 1200 mg infusions of eculizumab every other week with normal lab parameters. She is currently undergoing genetic testing at the University of Iowa laboratory to look for autoantibodies and genetic mutations. The response to eculizumab and the clinical course are typical for aHUS and diagnostic.

Table 1 aHUS Cases Associated with Inflammatory Bowel Disease

\begin{tabular}{|c|c|c|c|c|c|c|c|}
\hline Reference \# & $\mathrm{n}$ & Pt age & Pt gender & UC/CD & Creatinine improvement $(\mathrm{Y} / \mathrm{N})$ & TMA resolution $(\mathrm{Y} / \mathrm{N})$ & Ab/Mutation found? \\
\hline \multicolumn{8}{|l|}{ Case Reports } \\
\hline Beers [6] [abstract] & 1 & 19 & $\mathrm{~F}$ & $\mathrm{IBD}^{* *}$ & Y & Y & CFH Auto-Ab \& HZ CF1/CFH3 Del \\
\hline Webb [7] & 1 & 16 & M & UC & Y & Y & HZ MTHFR mutation ${ }^{* * *}$ \\
\hline Viada Bris $[8,9]^{*}$ & 1 & 15 & $\mathrm{~F}$ & UC & NT & NT & $\mathrm{HZ} C F H, H Z M C P$ \\
\hline Green H [10] & 1 & 27 & $\mathrm{~F}$ & UC & Y & Y & CFH Auto-Ab \\
\hline Hanna et.al. Case 1 & $(1 / 2)$ & 19 & M & $C D$ & Y & Y & No clear mutation found ${ }^{* * * *}$ \\
\hline Hanna et.al. Case 2 & $(2 / 2)$ & 49 & $F$ & UC & Y & Y & Testing not completed \\
\hline
\end{tabular}

$A b$ antibody, CD Crohn's disease, CF1 complement factor 1, CF3 complement factor $3, C F H$ complement factor $\mathrm{H}, H Z$ heterozygous, $M C P$ membrane cofactor protein, MTHFR methylenetetrahydrofolate reductase gene/protein product, $n$ number, $N$ no, NT not treated, Pt patient, UC ulcerative colitis, $Y$ yes. * 8 , 9 describe same case. ** IBD is only stated, it is not otherwise specified whether UC/CD.***Heterozygous MTHFR mutations are not generally thought to cause aHUS, therefore a known causative mutation was not found in Webb et.al. [7]. ****Early on in Case 1 a borderline positive C3 nephritic factor antibody was found and complement testing revealed a high level of soluble membrane attack complex 


\section{Discussion and conclusions}

We report a series of two patients with two variants of IBD presented with a very rare association with aHUS. The pathophysiology of aberrant complement activation does not require a specific trigger, and like rheumatological diseases, gastroenterological autoimmune disease like IBD can also trigger complement activation in susceptible individuals [1]. Interestingly, both patients had poorly controlled IBD at the time of aHUS diagnosis. The patients' IBD (CD case 1 , UC case 2 ), was eventually well controlled with intensive IBD therapy, alongside complement blockade for aHUS.

The lack of finding a complement mutation in patient 1 who had a relapsing course is suggestive of either: 1) a new complement mutation that has not been previously reported; 2) background gene effect. The two known classes of complement mutations are complement factor mutations resulting in auto-activation or increased activation of complement (factor $\mathrm{B}$, factor $\mathrm{I}$ ), and loss of function mutations resulting in decreased ability of selfcells to disarm complement proteins (like membrane cofactor protein, etc.) [11]. Other complement proteins interact with the complement cascade that when mutated can also affect complement function. For example, vitronectin is the newest genetic defect described that has been linked to aHUS $[11,12]$.

In addition to four other published case, we report the fifth and sixth cases of IBD associated aHUS successfully treated with eculizumab. Table 1 summarizes previous case reports in the literature and our current cases that highlight the infrequent association between IBD (CD and UC) and aHUS [6-10].

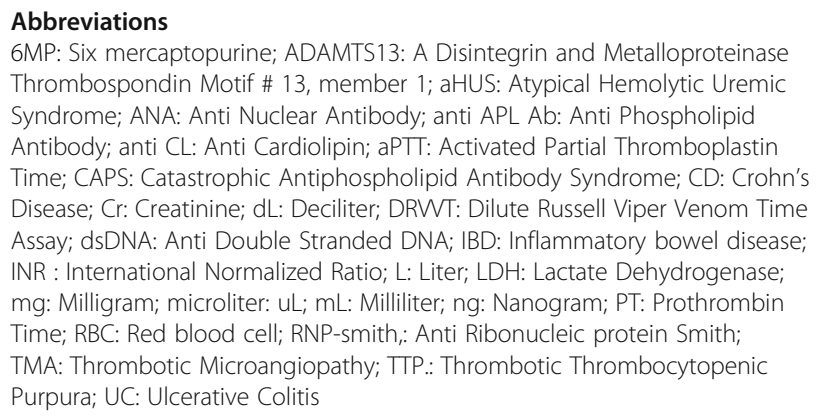

\section{Abbreviations}

6MP: Six mercaptopurine; ADAMTS13: A Disintegrin and Metalloproteinase Thrombospondin Motif \# 13, member 1; aHUS: Atypical Hemolytic Uremic Syndrome; ANA: Anti Nuclear Antibody; anti APL Ab: Anti Phospholipid Antibody; anti CL: Anti Cardiolipin; aPTT: Activated Partial Thromboplastin Time; CAPS: Catastrophic Antiphospholipid Antibody Syndrome; CD: Crohn's Disease; Cr: Creatinine; dL: Deciliter; DRWT: Dilute Russell Viper Venom Time Assay; dsDNA: Anti Double Stranded DNA; IBD: Inflammatory bowel disease; INR : International Normalized Ratio; L: Liter; LDH: Lactate Dehydrogenase; mg: Milligram; microliter: UL; mL: Milliliter; ng: Nanogram; PT: Prothrombin Time; RBC: Red blood cell; RNP-smith,: Anti Ribonucleic protein Smith; TMA: Thrombotic Microangiopathy; TTP.: Thrombotic Thrombocytopenic Purpura; UC: Ulcerative Colitis

\section{Acknowledgements}

IK is supported in part by funds from the NIH (R01-DK077162), the Allan Smidt Charitable Fund, the Factor Family Foundation, and the Ralph Block Family Foundation.

\section{Authors' contributions}

$\mathrm{RMH}$, directed manuscript writing as first author. NM, assisted in writing case 2 and revised text of manuscript. RB, assisted in the background and with case 2. LA, assisted in writing case 1. US, helped with case 1 and figures. BY, edited case with oversight of gastrointestinal portions of introduction and conclusion. PB, assisted in making figures. MF, edited text and compiled references. IK, edited text of manuscript as senior author. All authors read and approved the final manuscript.

\section{Authors' information}

Ramy M, Hanna MD FASN FACP Is an assistant Professor and nephrology consultant at UCLA David Geffen School of Medicine with expertise in complement mediated renal disorders.

Noah Merin MD PhD is an assistant professor and hematology / oncology consultant at Cedars Sinai Medical Center with expertise in complement mediated diseases.

Richard Burwick MD MPH is an associate professor and maternal fetal medicine consultant at Cedars Sinai Medical Center with key expertise in pre-eclampsia and complement mediated disorders during pregnancy. Ira Kurtz MD is a long term-NIH funded investigator, and the division chief of nephrology at UCLA.

\section{Funding}

IK is supported in part by funds from the NIH (R01-DK077162), the Allan Smidt Charitable Fund, the Factor Family Foundation and the Ralph Block Family Foundation.

Sponsorship: This work was not sponsored.

\section{Availability of data and materials}

Not applicable, no data.

\section{Ethics approval and consent to participate}

Not applicable, this was discussed with UCLA IRB and determined not to constitute human research material and not to need an institutional review board application.

\section{Consent for publication}

Consent for publication has been obtained in writing by authors from patients and has been documented in health record.

\section{Competing interests}

$\mathrm{RMH}, \mathrm{NM}$, and RB belong to the 2018 and 2019 Alexion Pharmaceuticals speaker's Bureau and received honoraria for speaking and consulting.

\section{Author details}

${ }^{1}$ Department of Medicine, Division of Nephrology, UCLA David Geffen School of Medicine, Room 7-155, Factor Bldg. 700 Tiverton Ave, Los Angeles, CA 90095, USA. ${ }^{2}$ Department of Medicine, Division of Nephrology, University of California Irvine School of Medicine, Irvine CA, USA. ${ }^{3}$ Department of Medicine, Division of Hematology Oncology, Cedars Sinai Medical Center, Los Angeles, USA. ${ }^{4}$ Department of Obstetrics and Gynecology, Division of Maternal Fetal Medicine, Cedars Sinai Medical Center, Los Angeles, USA. ${ }^{5}$ Department of Medicine, Division of Digestive Diseases, UCLA David Geffen School of Medicine, Los Angeles, USA. ${ }^{6}$ Department of Medicine, UCLA David Geffen School of Medicine, Los Angeles, USA. ${ }^{7}$ UCLA Brain Research Institute, Los Angeles, USA.

Received: 24 June 2019 Accepted: 31 July 2019

Published online: 09 September 2019

\section{References}

1. Hanna R, Marina B, Vandross A et.al. Atypical hemolytic uremic syndrome and complement blockade: established and emerging uses of complement activation. Curr Opin Nephrol Hypertens 2019, 28:000-000 DOl: https://doi. org/10.1097/MHN.0000000000000499.

2. Park MHCN, Ulmer S, Weitz IC. Complement-mediated thrombotic microangiopathy associated with lupus nephritis. Blood Advances. 2018;2: 2090-4.

3. Park MHWI. Effective treatment of thrombotic Microangiopathy associated with lupus nephritis with Eculizumab: a series of 8 cases. Blood. 2017;130:1043.

4. Hanna RMHM, Larson B, Lopez EA, Wilson J, Hendifar A. Thrombotic Microangiopathy due to catastrophic antiphospholipid antibody syndrome confirmed on skin biopsy and treated with. Eculizumab J Onco Nephrol. 2017;1:2.

5. Bianchi L, Gaiani F, Vincenzi F, et.al. Hemolytic uremic syndrome: differential diagnosis with the onset of inflammatory bowel diseases. Acta Biomed 2018;89(9-S):153-157. (Review).

6. Beers K, Zarrabi K, Yezina N, et al. Atypical HUS in a patient with inflammatory bowel disease. Am J Kidney Dis. 2017;69(4):A1-A105 Am J Kidney Dis. 2017;69(4): A1-A105. 
7. Webb TN, Griffiths H, Miyashita Y. Atypical hemolytic uremic syndrome and chronic ulcerative colitis treated with Eculizumab. Int J Med Pharm Case Reports Int J Med Pharm Case Reports. 2015;4(5):105-12.

8. Viada Bris JF, Velasco Rodríguez-Belvís M, de Lucas Collantes C. Et.al. Ulcerative colitis and atypical hemolytic-uremic syndrome: an unusual but potentially life-threating life complication. Inflamm Bowel Dis. 2018 Jun 20.

9. Viada Bris JF, Velasco Rodríguez-Belvís M, de Lucas Collantes C, et.al. Ulcerative Colitis and Atypical Hemolytic-Uremic Syndrome: An Unusual But Potentially Life-threatening Complication Inflamm Bowel Dis • Volume 00, Number 00, Month 2018 (letter to the editor).

10. Green $\mathrm{H}$, Harari E, Davidovits M, et al. Atypical HUS due to factor $\mathrm{H}$ antibodies in an adult patient successfully treated with eculizumab. Ren Fail. 2014;36(7):1119-21.

11. Rodriguez de Cordoba S, Hidalgo MS, Pinto S. Et.al. Genetics of atypical hemolytic uremic syndrome (aHUS). Semin Thromb Hemost. 2014;40(4):422-30.

12. Bu F, Zhang $Y$, Wang $K$, et.al. Genetic analysis of 400 patients refines understanding and implicates a new gene in atypical hemolytic uremic syndrome. J Am Soc Nephrol 2018;29(12):2809-2819.

\section{Publisher's Note}

Springer Nature remains neutral with regard to jurisdictional claims in published maps and institutional affiliations.

Ready to submit your research? Choose BMC and benefit from:

- fast, convenient online submission

- thorough peer review by experienced researchers in your field

- rapid publication on acceptance

- support for research data, including large and complex data types

- gold Open Access which fosters wider collaboration and increased citations

- maximum visibility for your research: over $100 \mathrm{M}$ website views per year

At BMC, research is always in progress.

Learn more biomedcentral.com/submissions 